\title{
Strong solutions for stochastic porous media equations with jumps
}

\author{
Viorel Barbu* Carlo Marinelli ${ }^{\dagger}$
}

15 February 2008

\begin{abstract}
We prove global well-posedness in the strong sense for stochastic generalized porous media equations driven by locally square integrable martingales with stationary independent increments.
\end{abstract}

\section{Introduction}

The purpose of this short note is to establish well-posedness in the strong sense for a class of nonlinear stochastic PDEs driven by Lévy noise. In particular, we shall consider the following stochastic porous media equation

$$
d X(t)-\Delta \beta(X(t)) d t=B(X(t-)) d M(t),
$$

where $M$ is a locally square integrable martingale with stationary independent increments taking values in a Hilbert space $K$, and the diffusion coefficient $B$ satisfies a Lipschitz assumption. Only minimal assumptions on the monotone function $\beta$ will be assumed. Full details on the data of the problem are given below. The deterministic counterpart of (1.1), which is often referred to as the generalized porous media equation, or the filtration equation (especially in the russian literature), has been extensively studied both for its physical importance and as a model nonlinear PDE (see e.g. the monograph [16] for a systematic treatment of the mathematical aspects).

Stochastic evolution equations of porous media type (with no polynomial growth assumptions on the monotone mapping $\beta$ ) such as (1.1) are an important class of nonlinear SPDE which cannot be studied by variational methods (see e.g. [9], [14], [15], [8], [12], [7]), nor by the semigroup approach (see e.g. [6], [5], [13]). Moreover, to the best of our knowledge, there seem to be very few results on general nonlinear SPDEs which do not fall in any of the above mentioned formulations. These simple observations provide the main motivation for studying such type of equations. Well-posedness for (1.1) has been established in 2 assuming that $M$ is an infinite dimensional Brownian motion and that $B$ is sufficiently regular.

In this paper we show that the results of 2 2 can be generalized to the case of discontinuous (in time) random forcing. Moreover, we prove that the solution of (1.1) lives in a "better" space than the one used in [2, and we introduce a concept of generalized solution that allows to remove some restrictions on the diffusion coefficient $B$.

${ }^{*}$ University Al. I. Cuza, 8 Blvd. Carol I, Iaşi 700506, Romania. E-mail vb41@uaic.ro

${ }^{\dagger}$ Institut für Angewandte Mathematik, Universität Bonn, Wegelerstr. 6, D-53115 Bonn, Germany. 


\section{Main result}

Let us begin introducing some notation. Given two separable Hilbert spaces $H, K$ we shall denote by $\mathcal{L}_{1}(H, K)$ and $\mathcal{L}_{2}(H, K)$ the space of trace-class and Hilbert-Schmidt operators, respectively, from $H$ to $K$. $\mathcal{L}_{1}^{+}$stands for the subset of $\mathcal{L}_{1}$ of positive operators. We shall write $\mathcal{L}_{1}(H)$ in place of $\mathcal{L}_{1}(H, H)$, and similarly for the other spaces. Given a self-adjoint operator $Q \in \mathcal{L}_{1}^{+}(H)$, we denote by $\mathcal{L}_{2}^{Q}$ the set of all (possibly unbounded) operators $B: Q^{1 / 2} H \rightarrow K$ such that $B Q^{1 / 2} \in \mathcal{L}_{2}(H, K)$. The norm in $\mathcal{L}_{2}^{Q}$ will be denoted by $|\cdot|_{Q}$. We shall denote by $C^{w}(I, X)$ the space of weakly continuous functions defined on the interval $I \subseteq \mathbb{R}$ and taking values in the Banach space $X$.

In order to state the result about existence and uniqueness of a strong solution to equation (1.1) we need to recall some facts from the theory of stochastic integration in Hilbert spaces. For all unexplained notation we refer to [10]. We shall denote by $\mathcal{M}_{l o c}^{2}(K)$ the space of locally square integrable martingales on $K$. Let $M \in \mathcal{M}_{l o c}^{2}(K)$ and $T>0$ a fixed (deterministic) time. The stopped process $M(t \wedge T)$ is an $L^{2}$-martingale, and recalling that $\left|M_{t}\right|_{K}^{2}$ is a submartingale, we have $\sup _{t>0} \mathbb{E}|M(t \wedge T)|_{K}^{2}=\mathbb{E}|M(T)|_{K}^{2}<\infty$, i.e. $M(t \wedge T) \in \mathcal{M}_{\infty}^{2}(K)$. We are therefore in the framework of [10], sect. 22, which allows to construct stochastic integrals of the type

$$
G \cdot M(t):=\int_{(0, t]} G(s) d M(s), \quad t \in[0, T],
$$

for a class of operator valued predictable processes $G$. In particular, according to the results of [10], [11], there exists a predictable $\mathcal{L}_{1}^{+}(K)$-valued process $\left(Q_{M}(t)\right)_{t \in[0, T]}$ such that

$$
\langle\langle M\rangle\rangle(t)=\int_{0}^{t} Q_{M}(s) d\langle M\rangle(s), \quad t \in[0, T],
$$

and the stochastic integral (2.1) is well defined for all predictable processes $G:[0, T] \times$ $\Omega \rightarrow \mathcal{L}_{2}^{Q_{M}}(K, H)$ such that

$$
\mathbb{E} \int_{0}^{t}\left|G(s) Q_{M}(s)^{1 / 2}\right|_{\mathcal{L}_{2}(K, H)}^{2} d\langle M\rangle(s)<\infty
$$

The set of all such processes will be denoted by $\mathcal{G}(H)$. Here and everywhere in the following we write, with a slight abuse of notation, $\int_{0}^{t}$ instead of $\int_{(0, t]}$.

Remark 2.1. If $M$ has stationary independent increments (i.e. $M$ is also a Lévy process), then $Q_{M}=(\operatorname{Tr} Q)^{-1} Q$, where $Q$ is the covariance operator of $M$, and $\langle M\rangle(t)=t \operatorname{Tr} Q$, $\langle\langle M\rangle\rangle(t)=t(\operatorname{Tr} Q)^{-1} Q$ for all $t \in[0, T]$. Moreover, $Q$ is a deterministic operator.

We shall now state precisely the stochastic equation we shall deal with, together with some more notation. Let $\Xi$ be an open bounded subset of $\mathbb{R}^{d}, d>1$, with smooth boundary $\partial \Xi$, and define, for $t>0$,

$$
Q_{t}=(0, t) \times \Xi, \quad \partial Q_{t}=(0, t) \times \partial \Xi .
$$

We denote by $H^{-1}:=H^{-1}(\Xi)$ the dual of the Sobolev space $H_{0}^{1}(\Xi)$, endowed with the scalar product $\langle f, g\rangle_{-1}=\left\langle(-\Delta)^{-1} f, g\right\rangle$, where $\langle\cdot, \cdot\rangle$ is the duality pairing between $H_{0}^{1}(\Xi)$ and $H^{-1}(\Xi)$, and $\Delta$ stands for the Laplacian with Dirichlet homogeneous boundary conditions. The inner product in $L^{2}(\Xi)$ will be denoted by $\langle\cdot, \cdot\rangle_{2}$. Whenever no misunderstanding can arise, we shall write all functional spaces without explicitly indicating the domain $\Xi$, e.g. $H^{-1}=H^{-1}(\Xi)$, etc. 
We shall study existence, uniqueness and regular dependence on the initial datum for the following stochastic Cauchy problem:

$$
\begin{cases}d X(t)-\Delta \beta(X(t)) d t=B(X(t-)) d M(t) & \text { in } Q_{T} \\ \beta(X(t))=0 & \text { in } \partial Q_{T} \\ X(0)=x & \text { in } \Xi\end{cases}
$$

under a set of assumptions on $\beta, B$ and $M$. In particular, we shall assume that the diffusion coefficient is of the form

$$
B: H^{-1} \rightarrow \mathcal{L}_{2}^{Q}\left(K, D\left((-\Delta)^{\gamma}\right), \quad \gamma>d / 2,\right.
$$

and satisfies

$$
|B(x)|_{Q}^{2} \leq k\left(1+|x|^{2}\right), \quad|B(x)-B(y)|_{Q}^{2} \leq k|x-y|^{2},
$$

for some constant $k>0$.

The (multivalued) function $\beta: \mathbb{R} \rightarrow 2^{\mathbb{R}}$ is assumed to be a maximal monotone graph in $\mathbb{R} \times \mathbb{R}$ such that $0 \in \beta(0), D(\beta)=R(\beta)=\mathbb{R}$, and

$$
\limsup _{|x| \rightarrow \infty} \frac{j(-x)}{j(x)}<\infty
$$

where $j: \mathbb{R} \rightarrow \mathbb{R}$ is a convex function such that $\beta=\partial j$ (such a function always exists and is unique modulo addition of constants - see e.g. [1], [4]). Here $\partial$ stands for the subdifferential in the sense of convex analysis.

Definition 2.2. An adapted weakly càdlàg process $X \in L^{1}((0, T) \times \Xi \times \Omega)$ is a strong solution of (1.1) if there exists an adapted process $\eta \in L^{1}((0, T) \times \Xi \times \Omega)$ such that $\eta(t, \xi) \in \beta(X(t, \xi))$ a.e. in $Q_{T}, \mathbb{P}$-a.s.,

$$
\begin{gathered}
t \mapsto \int_{0}^{t} \eta(s, \xi) d s \in C^{w}\left([0, T], H_{0}^{1}\right), \\
X(t)-\Delta \int_{0}^{t} \eta(s) d s=x+\int_{0}^{t} B(X(s-)) d M(s), \quad \forall t \in[0, T], \mathbb{P}-\text { a.s. }
\end{gathered}
$$

and $j(X), j^{*}(\eta) \in L^{1}((0, T) \times \Xi \times \Omega)$.

We first establish a well-posedness result for the SPDE with additive noise. Let us denote by $\mathcal{H}_{2}(T)$ and $\mathbb{H}_{2}(T)$ the spaces of adapted processes $u:[0, T] \rightarrow H^{-1}$ such that

$$
\sup _{t \leq T} \mathbb{E}|u(t)|_{-1}^{2}<\infty \quad \text { and } \quad \mathbb{E} \sup _{t \leq T}|u(t)|_{-1}^{2}<\infty
$$

respectively. We shall also denote by $\mathcal{H}_{2}$ the space of $H^{-1}$-valued random variables with finite second moment.

Theorem 2.3. If $G \in \mathcal{G}\left(D\left((-\Delta)^{\gamma}\right)\right), \gamma>d / 2$, then for each $x \in \mathcal{H}_{2}$ there exists a unique strong solution to the equation

$$
\begin{cases}d Y(t)-\Delta \beta(Y(t)) d t=G(t) d M(t) & \text { in } Q_{T} \\ \beta(Y(t))=0 & \text { in } \partial Q_{T} \\ Y(0)=x & \text { in } \Xi\end{cases}
$$


Moreover, for $G_{1}, G_{2} \in \mathcal{G}\left(D\left((-\Delta)^{\gamma}\right)\right)$ and $y_{1}, y_{2} \in \mathcal{H}_{2}$, denoting by $Y\left(t, y_{i}, G_{i}\right), i=1,2$, the solutions of (2.8) with $G=G_{i}$ and $Y(0)=y_{i}$, respectively, the following estimate holds:

$$
\mathbb{E}\left|Y\left(t, y_{1}, G_{1}\right)-Y\left(t, y_{2}, G_{2}\right)\right|_{-1}^{2} \leq \mathbb{E}\left|y_{1}-y_{2}\right|_{-1}^{2}+\mathbb{E} \int_{0}^{t}\left|G_{1}(s)-G_{2}(s)\right|_{Q_{M}}^{2} d\langle M\rangle(s) .
$$

Finally, the solution map $x \mapsto Y$ is a contraction from $\mathcal{H}_{2}$ to $\mathbb{H}_{2}(T)$.

Our main result is the following.

Theorem 2.4. Assume that $M$ has stationary independent increments. Then for each $x \in \mathcal{H}_{2}$ there exists a unique strong solution of (2.3). Moreover, the solution map $x \mapsto X$ is Lipschitz from $\mathcal{H}_{2}$ to $\mathbb{H}_{2}(T)$.

Remark 2.5. Note that in theorem 2.3 the process $M$ is only assumed to belong to $\mathcal{M}_{\text {loc }}^{2}(K)$, i.e. we do not need to assume that it has stationary independent increments as in theorem 2.4 .

\section{Auxiliary results}

Since the stochastic integral (2.1) is a locally square integrable martingale for any $G$ satisfying (2.2), Doob's inequality yields the following simple result.

Lemma 3.1. Let $M \in \mathcal{M}_{\text {loc }}^{2}(K)$ and $G \in \mathcal{G}(H)$. Then

$$
\mathbb{P}\left(\sup _{t \leq T}|G \cdot M(t)|_{H}<\infty\right)=1
$$

Proof. It is enough to note that, by Cauchy-Schwartz' inequality,

$$
\mathbb{E} \sup _{t \leq T}|G \cdot M(t)|_{H} \leq\left(\mathbb{E} \sup _{t \leq T}|G \cdot M(t)|_{H}^{2}\right)^{1 / 2},
$$

and that (since $|G \cdot M|_{H}^{2}$ is a submartingale) Doob's inequality yields

$$
\mathbb{E} \sup _{t \leq T}|G \cdot M(t)|_{H}^{2} \leq 4 \mathbb{E}|G \cdot M(T)|_{H}^{2}=4 \mathbb{E} \int_{0}^{T}|G(s)|_{Q_{M}(s)}^{2} d\langle M\rangle(s)<\infty .
$$

The last step follows by the isometric formula (see [11]) and (2.2).

We shall also need an Itô formula for the square of the norm of strong solutions to (2.8).

Lemma 3.2. Let $Y$ be a strong solution of (2.8). Then one has

$$
\begin{aligned}
|Y(t)|_{-1}^{2}= & |Y(0)|_{-1}^{2}-2 \int_{0}^{t}\langle Y(s), \eta(s)\rangle_{2} d s \\
& +2 \int_{0}^{t}\langle Y(s-), G(s) d M(s)\rangle_{-1}+[G \cdot M](s)
\end{aligned}
$$

for all $t \in[0, T], \mathbb{P}$-a.s. 
Proof. Let us set, for $m \in \mathbb{N}$ sufficiently large,

$$
\begin{aligned}
Y_{\varepsilon}(t) & =(1-\varepsilon \Delta)^{-m} Y(t), & & \eta_{\varepsilon}=(1-\varepsilon \Delta)^{-m} \eta(t), \\
G_{\varepsilon}(t) & =(1-\varepsilon \Delta)^{-m} G(t), & & x_{\varepsilon}=(1-\varepsilon \Delta)^{-m} Y(0) .
\end{aligned}
$$

Then we have by (2.8) that

$$
d Y_{\varepsilon}(t)=\Delta \eta_{\varepsilon}(t) d t+G_{\varepsilon}(t) d M(t), \quad Y_{\varepsilon}(0)=x_{\varepsilon}, \quad \eta_{\varepsilon}=0 \text { on } \partial \Xi
$$

and Itô's formula for $\left|Y_{\varepsilon}(t)\right|_{-1}^{2}$ yields

$$
\begin{aligned}
\left|Y_{\varepsilon}(t)\right|_{-1}^{2}= & \left|x_{\varepsilon}\right|_{-1}^{2}-2 \int_{0}^{t}\left\langle Y_{\varepsilon}(s-), \eta_{\varepsilon}(s)\right\rangle_{2} d s+2 \int_{0}^{t}\left\langle Y_{\varepsilon}(s-), G_{\varepsilon}(s) d M(s)\right\rangle_{-1} \\
& +\int_{0}^{t} d \operatorname{Tr} \llbracket G_{\varepsilon} \cdot M \rrbracket^{c}(s)+\sum_{s \leq t}\left|\Delta\left(G_{\varepsilon} \cdot M\right)(s)\right|_{-1}^{2} \\
= & \left|x_{\varepsilon}\right|_{-1}^{2}-2 \int_{0}^{t}\left\langle Y_{\varepsilon}(s-), \eta_{\varepsilon}(s)\right\rangle_{2} d s+2 \int_{0}^{t}\left\langle Y_{\varepsilon}(s-), G_{\varepsilon}(s) d M(s)\right\rangle_{-1} \\
& +\left[G_{\varepsilon} \cdot M\right](t)
\end{aligned}
$$

$\mathbb{P}$-a.s., where we have used the identity $\operatorname{Tr} \llbracket Z \rrbracket=[Z]$, which holds for any semimartingale $Z$. We clearly have $\left|x_{\varepsilon}\right|_{-1}^{2} \rightarrow\left|Y_{0}\right|_{-1}^{2} \mathbb{P}$-a.s. as $\varepsilon \rightarrow 0$. Moreover, we have

$$
\int_{0}^{t}\left\langle Y_{\varepsilon}(s-), \eta_{\varepsilon}(s)\right\rangle_{2} d s=\int_{0}^{t}\left\langle Y_{\varepsilon}(s), \eta_{\varepsilon}(s)\right\rangle_{2} d s \rightarrow \int_{0}^{t}\langle Y(s), \eta(s)\rangle_{2} d s
$$

as it follows from lemma 3.1 of [2]. In fact, recalling that $Y_{\varepsilon}(s)=y_{\varepsilon}(s)+G_{\varepsilon} \cdot M(s)$, where $y_{\varepsilon}$ is weakly continuous and $G_{\varepsilon} \cdot M$ is càdlàg, we have

$$
\int_{0}^{t}\left\langle y_{\varepsilon}(s-), \eta_{\varepsilon}(s)\right\rangle_{2} d s=\int_{0}^{t}\left\langle y_{\varepsilon}(s), \eta_{\varepsilon}(s)\right\rangle_{2} d s
$$

by weak continuity of $y_{\varepsilon}$, and

$$
\int_{0}^{t}\left\langle G_{\varepsilon} \cdot M(s)-G_{\varepsilon} \cdot M(s-), \eta_{\varepsilon}(s)\right\rangle_{2} d s=0
$$

because the times of discontinuity of càdlàg processes are at most countable, hence with Lebesgue measure zero.

Let us now consider the third term on the right hand side of (3.2). We can write

$$
\begin{aligned}
& \mathbb{E}\left|\int_{0}^{t}\left\langle Y_{\varepsilon}(s-), G_{\varepsilon}(s) d M(s)\right\rangle_{-1}-\int_{0}^{t}\langle Y(s-), G(s) d M(s)\rangle_{-1}\right|^{2} \\
& \leq \mathbb{E}\left|\int_{0}^{t}\left\langle Y_{\varepsilon}(s-),\left(G_{\varepsilon}(s)-G(s)\right) d M(s)\right\rangle_{-1}\right|^{2} \\
& \quad+\mathbb{E}\left|\int_{0}^{t}\left\langle Y_{\varepsilon}(s-)-Y(s-), G_{\varepsilon}(s) d M(s)\right\rangle_{-1}\right|^{2} \\
& \leq \mathbb{E} \int_{0}^{t}|Y(s-)|_{-1}^{2}\left|G(s)-G_{\varepsilon}(s)\right|_{Q_{M}}^{2} d\langle M\rangle(s) \\
& \quad+\mathbb{E} \int_{0}^{t}\left|Y(s-)-Y_{\varepsilon}(s-)\right|_{-1}^{2}|G(s)|_{Q_{M}}^{2} d\langle M\rangle(s) \rightarrow 0
\end{aligned}
$$


thus

$$
\int_{0}^{t}\left\langle Y_{\varepsilon}(s-), G_{\varepsilon}(s) d M(s)\right\rangle_{-1} \rightarrow \int_{0}^{t}\langle Y(s-), G(s) d M(s)\rangle_{-1}
$$

$\mathbb{P}$-a.s. and for all $t \in[0, T]$, at least on a subsequence of $\varepsilon$, still denoted by $\varepsilon$, as $\varepsilon \rightarrow 0$. Similarly, for the last term on the right hand side of (3.2) we can write, recalling that $G_{\varepsilon} \cdot M \in \mathcal{M}_{l o c}^{2}(H)$

$\mathbb{E}\left[G_{\varepsilon} \cdot M\right](t)=\mathbb{E}\left\langle G_{\varepsilon} \cdot M\right\rangle(t) \leq \mathbb{E} \int_{0}^{t}\left|G_{\varepsilon}(s)\right|_{Q_{M}}^{2} d\langle M\rangle(s) \leq \mathbb{E} \int_{0}^{t}|G(s)|_{Q_{M}}^{2} d\langle M\rangle(s)<\infty$,

hence by monotone convergence we have $\mathbb{E}\left[G_{\varepsilon} \cdot M\right](t) \rightarrow \mathbb{E}[G \cdot M](t)$, and $\left[G_{\varepsilon} \cdot M\right](t) \rightarrow$ $[G \cdot M](t) \mathbb{P}$-a.s. for all $t \in[0, T]$, passing to a subsequence if necessary.

\section{Proof of theorem 2.3}

We follow the strategy of proof of [2], and we limit ourselves to a sketch of proof underlining the relevant differences.

Let us consider the approximating SPDE (in integral form)

$$
X(t)=x+\int_{0}^{t} \Delta\left(\beta_{\lambda}(X(s))+\lambda X(s)\right) d s+G \cdot M(t) .
$$

Then one has the following result.

Lemma 4.1. The SPDE (4.1) admits a unique càdlàg adapted solution $X_{\lambda}$ such that

$$
X_{\lambda}, \beta_{\lambda}\left(X_{\lambda}\right) \in L^{2}\left([0, T], H_{0}^{1}\right) .
$$

Proof. Equation (4.1) can be equivalently rewritten as the deterministic PDE with random coefficients

$$
y^{\prime}=\Delta \tilde{\beta}_{\lambda}(y+G \cdot M),
$$

setting $y=X-G \cdot M$ and $\tilde{\beta}_{\lambda}(x):=\beta_{\lambda}(x)+\lambda x$. Moreover, for any fixed $\omega \in \Omega$, the time-dependent operator

$$
\begin{aligned}
\mathcal{A}(t): H_{0}^{1} & \rightarrow H^{-1} \\
x & \mapsto-\Delta \tilde{\beta}_{\lambda}(x+G \cdot M)
\end{aligned}
$$

satisfies the assumptions of Theorem III.4.2 in [1], hence (4.2) admits a unique solution

$$
y_{\lambda} \in C\left([0, T], L^{2}\right) \cap L^{2}\left([0, T], H_{0}^{1}\right),
$$

with $y_{\lambda}^{\prime} \in L^{2}\left([0, T], H^{-1}\right)$. Moreover, $y_{\lambda}$ depends continuously on $G \cdot M$ with respect to pathwise convergence in $H^{-1}$, hence $X_{\lambda}:=y_{\lambda}+G \cdot M$ is an adapted càdlàg solution of (4.11), as required.

We shall need some a priori estimates for $z_{\lambda}$ and $\eta_{\lambda}$, where

$$
z_{\lambda}=(1+\lambda \beta)^{-1} X_{\lambda}, \quad \eta_{\lambda}=\beta_{\lambda}\left(X_{\lambda}\right) .
$$


Lemma 4.2. There exists $\Omega_{0} \subset \Omega$ with $\mathbb{P}\left(\Omega_{0}\right)=1$ such that, for every fixed $\omega \in \Omega_{0}$, one has

$$
\begin{gathered}
\int_{Q_{T}}\left(j\left(z_{\lambda}\right)+j^{*}\left(\eta_{\lambda}\right)\right) d \xi d s \leq N_{1}\left(1+|x|_{-1}^{2}\right), \\
\int_{Q_{T}}\left|X_{\lambda}-z_{\lambda}\right|^{2} d \xi d s \leq 2 \lambda N_{1}\left(1+|x|_{-1}^{2}\right),
\end{gathered}
$$

where $N_{1}$ is a positive constant that may depend on $\omega$.

Proof. It can be repeated almost word for word as in section 3.1 of [2], with the following modifications. By lemma 3.1. Sobolev's embedding theorem $D\left((-\Delta)^{\gamma}\right) \subset L^{\infty}, \gamma>d / 2$, and the hypothesis that $\mathbb{E}|x|_{-1}^{2}<\infty$, it follows that there exists $\Omega_{0} \subseteq \Omega, \mathbb{P}\left(\Omega_{0}\right)=1$, such that

$$
\sup _{t \leq T}|G \cdot M(t)|_{L^{\infty}}<\infty, \quad|x(\omega)|_{-1}^{2}<\infty \quad \forall \omega \in \Omega_{0} .
$$

Using this estimate in place of the corresponding one for $W_{G}$ in [2], the claim follows.

The above estimates allow us to pass to the limit as $\lambda \rightarrow 0$, obtaining the following result.

Lemma 4.3. There exist $y \in C^{w}\left([0, T], H^{-1}\right) \cap L^{1}\left(Q_{T}\right)$ and $\eta \in L^{1}\left(Q_{T}\right) \cap L^{\infty}\left([0, T], H_{0}^{1}\right)$ such that

$$
y(t)+A \int_{0}^{t} \eta(s) d s=x .
$$

Proof. The hypotheses on $\beta$ imply that

$$
\lim _{|u| \rightarrow \infty} j(u) /|u|=\lim _{|p| \rightarrow \infty} j^{*}(p) /|p|=+\infty,
$$

which together with (4.3) yields that $z_{\lambda}$ and $\eta_{\lambda}$ are bounded and equiintegrable, hence weakly compact in $L^{1}\left(Q_{T}\right)$, as follows by the Dunford-Pettis criterion. Therefore, on a subsequence again denoted by $\lambda$, we have, by the same arguments of [2],

$$
\begin{aligned}
z_{\lambda} & \rightarrow z & & \text { in } L^{1}\left(Q_{T}\right) \\
\eta_{\lambda} & \rightarrow \eta & & \text { in } L^{1}\left(Q_{T}\right) \\
y_{\lambda} & \rightarrow y & & \text { in } L^{1}\left(Q_{T}\right) \\
y_{\lambda}(t) & \rightarrow y(t) & & \text { in } H \quad \forall t \in[0, T] \\
y_{\lambda} & \stackrel{*}{\rightarrow} & & \text { in } L^{\infty}\left([0, T], H_{0}^{1}\right) \\
\int_{0}^{.}\left(\eta_{\lambda}(s)+\lambda y_{\lambda}(s)\right) d s & \stackrel{*}{\rightarrow} \int_{0} \eta(s) d s & & \text { in } L^{\infty}\left([0, T], H_{0}^{1}\right) .
\end{aligned}
$$

where $y$ and $\eta$ satisfy (4.5).

One then continues proving that $\eta \in \beta(y+G \cdot M)$ a.e. in $Q_{T}$, and that such $y$ and $\eta$ are unique. Hence the above convergence results hold $\mathbb{P}$-a.s. for any choice of the sequence $\lambda$. In particular, $y$ and $\eta$ are adapted processes. Moreover, since $G \cdot M$ is càdlàg and $y$ is weakly continous, it follows that $Y(t)=y(t)+G \cdot M(t)$ is an $H^{-1}$-valued weakly càdlàg process such that

$$
Y(t)-\Delta \int_{0}^{t} \eta(s) d s=x+G \cdot M(t) \quad \forall t \in[0, T] \quad \mathbb{P} \text {-a.s. }
$$

i.e. $Y$ solves $(2.8)$. 
Once existence has been established, we need to prove uniqueness and continuous dependence on the initial datum. This can be achieved with the help of Lemma 3.2. In particular, taking into account that the second term on the right hand side of (3.1) is negative because $\eta(s) \in \beta(Y(s)) \mathbb{P}$-a.s. for a.a. $s \in[0, T]$, we have, by Lemma 3.2,

$$
\begin{aligned}
\left|Y_{1}(t)-Y_{2}(t)\right|_{-1}^{2} \leq & \left|y_{1}-y_{2}\right|_{-1}^{2}+2 \int_{0}^{t}\left\langle Y_{1}(s-)-Y_{2}(s-),\left(G_{1}(s)-G_{2}(s)\right) d M(s)\right\rangle_{-1} \\
& +\left[\left(G_{1}-G_{2}\right) \cdot M\right](t),
\end{aligned}
$$

where we set, for simplicity of notation, $Y_{i}:=Y\left(\cdot, y_{i}, G_{i}\right), i=1,2$. Taking expectation on both sides, we are left with

$$
\mathbb{E}\left|Y_{1}(t)-Y_{2}(t)\right|_{-1}^{2} \leq \mathbb{E}\left|y_{1}-y_{2}\right|_{-1}^{2}+\mathbb{E} \int_{0}^{t} \mid\left(G_{1}(s)-\left.G_{2}(s)\right|_{Q_{M}} ^{2} d\langle M\rangle(s) .\right.
$$

Similarly, if $G_{1}=G_{2}$, (4.6) immediately yields

$$
\mathbb{E} \sup _{t \leq T}\left|Y\left(t, y_{1}\right)-Y\left(t, y_{2}\right)\right|_{-1}^{2} \leq \mathbb{E}\left|y_{1}-y_{2}\right|_{-1}^{2} .
$$

\section{Proof of theorem 2.4}

Consider the equation

$$
d Y(t)=\Delta \beta(Y(t)) d t+B(X(t-)) d M(t), \quad t \in[0, T],
$$

and define the operator $\Phi: X \mapsto Y$ that associates to $X \in \mathbb{H}_{2}(T)$ the solution $Y$ of (5.1). We are going to prove that $\Phi$ maps $\mathbb{H}_{2}(T)$ to $\mathbb{H}_{2}(T)$ and it is a contraction. Moreover, since $t \mapsto B(X(t-))$ is predictable, we know by theorem 2.3 that $Y$ is adapted and weakly càdlàg. Let us first obtain two estimates that hold for any quasi-left-continuous $M \in \mathcal{M}_{l o c}^{2}(K)$. Itô's formula yields

$$
|Y(t)|_{-1}^{2}+2 \int_{0}^{t}\langle Y(s), \eta(s)\rangle_{2} d s=2 \int_{0}^{t}\langle Y(s-), B(X(s-)) d M(s)\rangle_{-1}+\left[B\left(X_{-}\right) \cdot M\right](t)
$$

Since $\langle Y(s), \eta(s)\rangle \geq 0 \mathbb{P}$-a.s. for all $s \leq t$, we can write

$$
\mathbb{E} \sup _{t \leq T}|Y(t)|_{-1}^{2} \leq 2 \mathbb{E} \sup _{t \leq T} \mid \int_{0}^{t}\left\langle Y(s-),(B(X(s-)) d M(s)\rangle_{-1}\left|+\mathbb{E} \int_{0}^{T}\right| B\left(\left.X(s)\right|_{Q_{M}} ^{2} d\langle M\rangle(s),\right.\right.
$$

where we have used the relations

$$
\begin{aligned}
\mathbb{E} \sup _{t \leq T}\left[B\left(X_{-}\right) \cdot M\right](t) & =\mathbb{E}\left[B\left(X_{-}\right) \cdot M\right](T)=\mathbb{E}\left\langle B\left(X_{-}\right) \cdot M\right\rangle(T) \\
& \leq \mathbb{E} \int_{0}^{T} \mid B\left(\left.X(s)\right|_{Q} ^{2} d\langle M\rangle(s),\right.
\end{aligned}
$$

and $B\left(X_{-}\right)$stands for $t \mapsto B(X(t-))$. Setting $Z=B\left(X_{-}\right) \cdot M$, Davis' inequality and the elementary inequality $a b \leq \varepsilon a^{2}+\frac{b^{2}}{\varepsilon}$ yield

$$
\begin{aligned}
& \mathbb{E} \sup _{t \leq T} \mid \int_{0}^{t}\left\langle Y(s-),(B(X(s-)) d M(s)\rangle_{-1}\left|=\mathbb{E} \sup _{t \leq T}\right| \int_{0}^{t}\langle Y(s-), d Z(s)\rangle_{-1}\right| \\
& \quad \leq 3 \mathbb{E}\left[Y_{-} \cdot Z\right](T)^{1 / 2} \leq 3 \mathbb{E} \sup _{t \leq T}|Y(t)|_{-1}[Z](T)^{1 / 2} \\
& \quad \leq 3 \varepsilon \mathbb{E} \sup _{t \leq T}|Y(s)|_{-1}^{2}+\frac{3}{\varepsilon} \mathbb{E} \int_{0}^{T} \mid B\left(\left.X(s)\right|_{Q_{M}} ^{2} d\langle M\rangle(s),\right.
\end{aligned}
$$


where we have used again

$$
\mathbb{E}[Z](T)=\mathbb{E}\langle Z\rangle(T) \leq \mathbb{E} \int_{0}^{T} \mid B\left(\left.X(s)\right|_{Q_{M}} ^{2} d\langle M\rangle(s) .\right.
$$

Thus we obtain

$$
(1-6 \varepsilon)|Y|_{\mathbb{H}_{2}(T)}^{2} \leq(6 / \varepsilon+1) \mathbb{E} \int_{0}^{T}|B(X(s))|_{Q_{M}}^{2} d\langle M\rangle(s) .
$$

Similarly, writing

$$
\left\{\begin{array}{l}
d Y_{1}=\Delta \beta\left(Y_{1}\right) d t+B\left(X_{1-}\right) d M \\
d Y_{2}=\Delta \beta\left(Y_{2}\right) d t+B\left(X_{2-}\right) d M,
\end{array}\right.
$$

with $Y_{1}(0)=Y_{2}(0)$, using again Itô's formula (see lemma [3.2), in complete analogy to the above derivation, we obtain the estimate

$$
(1-6 \varepsilon)\left|Y_{1}-Y_{2}\right|_{\mathbb{H}_{2}(T)}^{2} \leq(6 / \varepsilon+1) \mathbb{E} \int_{0}^{T}\left|B\left(X_{1}(t)\right)-B\left(X_{2}(t)\right)\right|_{Q_{M}}^{2} d\langle M\rangle(t) .
$$

If $M \in \mathcal{M}_{\text {loc }}^{2}(K)$ has also stationary independent increments, then, in view of remark 2.1. we have

$$
\begin{aligned}
\mathbb{E} \int_{0}^{T}|B(X(s))|_{Q_{M}}^{2} d\langle M\rangle(s) & =\mathbb{E} \int_{0}^{T}|B(X(s))|_{Q}^{2} d s \\
& \leq k \mathbb{E} \int_{0}^{T}\left(1+|X(s)|^{2}\right) d s \\
& \leq k T\left(1+|X|_{\mathbb{H}_{2}(T)}^{2}\right)<\infty,
\end{aligned}
$$

hence, choosing $\varepsilon<1 / 6,|Y|_{\mathbb{H}_{2}(T)}^{2}<\infty$, by virtue of (5.3). This proves that the image of $\Phi$ is contained in $\mathbb{H}_{2}(T)$. Let us now show that $\Phi$ is a contraction. In fact, (5.4) and assumption (2.5) yield

$$
\left|Y_{1}-Y_{2}\right|_{\mathbb{H}_{2}(T)}^{2} \leq \frac{1+6 / \varepsilon}{1-6 \varepsilon} k T\left|X_{1}-X_{2}\right|_{\mathbb{H}_{2}(T)}^{2},
$$

i.e. $\Phi$ is a contraction on $\mathbb{H}_{2}(T)$ whenever

$$
T<\frac{1-6 \varepsilon}{1+6 / \varepsilon} \frac{1}{k} .
$$

Then, by Banach fixed point theorem, there exists a unique solution of (1.1). If $T$ does not satisfy (5.5), then one proceeds in a classical way considering intervals $\left[0, T_{0}\right]$, $\left[T_{0}, 2 T_{0}\right]$, etc., with suitably small $T_{0}$, such that $\Phi$ is a contraction on $\mathbb{H}_{2}\left(T_{0}\right)$.

In order to prove Lipschitz continuity of the solution map, note that we have

$$
(1-6 \varepsilon)\left|Y\left(\cdot, y_{1}\right)-Y\left(\cdot, y_{2}\right)\right|_{\mathbb{H}_{2}(T)}^{2} \leq(6 / \varepsilon+1) k T\left|Y\left(\cdot, y_{1}\right)-Y\left(\cdot, y_{2}\right)\right|_{\mathbb{H}_{2}(T)}^{2}+\left|y_{1}-y_{2}\right|_{\mathcal{H}_{2}}^{2},
$$

hence for any $T_{0}$ such that

$$
1-6 \varepsilon-k T_{0}(6 / \varepsilon+1)>0
$$

we have

$$
\left|Y\left(\cdot, y_{1}\right)-Y\left(\cdot, y_{2}\right)\right|_{\mathbb{H}_{2}\left(T_{0}\right)} \leq N_{0}\left|y_{1}-y_{2}\right|_{\mathcal{H}_{2}},
$$

where

$$
N_{0}=\left(1-6 \varepsilon-k T_{0}(6 / \varepsilon+1)\right)^{-1 / 2}
$$

Considering intervals of length $T_{0}$ covering $[0, T]$ one finally gets

$$
\left|Y\left(\cdot, y_{1}\right)-Y\left(\cdot, y_{2}\right)\right|_{\mathbb{H}_{2}(T)} \leq N\left|y_{1}-y_{2}\right|_{\mathcal{H}_{2}},
$$

where $N=N(k, T)$. 


\section{Generalized solutions}

In this section we introduce a concept of generalized solution for equation (1.1), which allows to replace the assumption (2.4) with

$$
B: H^{-1} \rightarrow \mathcal{L}_{2}^{Q}\left(K, H^{-1}\right) .
$$

The definition of generalized solution is clearly inspired by the corresponding one in the theory of PDEs associated to maximal monotone operators - see e.g. [3, 4]. As we did before, we start with the case of additive noise and general $M \in \mathcal{M}_{\text {loc }}^{2}(K)$.

Definition 6.1. Let $G \in \mathcal{G}\left(H^{-1}\right)$. An adapted process $Y$ is called a $\mathcal{H}$-generalized solution of (2.8) if there exists a sequence $\left\{G_{n}\right\}_{n \in \mathbb{N}} \subset \mathcal{G}\left(D\left((-\Delta)^{\gamma}\right)\right)$ with

$$
\lim _{n \rightarrow \infty} \mathbb{E} \int_{0}^{T}\left|G_{n}(s)-G(s)\right|_{Q_{M}}^{2} d\langle M\rangle(s)=0
$$

such that the solution $Y_{n}$ to

$$
d Y(t)=\Delta \beta(Y(t)) d t+G_{n}(t) d M(t)
$$

equipped with the same initial and boundary conditions of (2.8), converges to $Y$ in $\mathcal{H}_{2}(T)$. If the convergence is in $\mathbb{H}_{2}(T), X$ is called $\mathbb{H}$-generalized solution.

It is clear that a $\mathbb{H}$-generalized solution is also a $\mathcal{H}$-generalized solution. In the following we shall refer to $\mathbb{H}$-generalized solutions simply as generalized solutions.

Theorem 6.2. Let $G \in \mathcal{G}\left(H^{-1}\right)$. Then (2.8) admits a unique generalized solution. Moreover, the solution map $x \mapsto Y$ is a contraction from $\mathcal{H}_{2}$ to $\mathbb{H}_{2}(T)$.

For the proof of the theorem we need the following approximation procedure for elements of the space $H^{-1}$. Let $f \in H^{-1}$. Then there exists $F \in H_{0}^{1}$ such that $f=\Delta F$, and $|f|_{-1}^{2}=|\nabla F|_{2}^{2}$. Set $F_{n}=\zeta_{n} * F$ and $f_{n}=\Delta F_{n}$, where $\left\{\zeta_{n}\right\}_{n \in \mathbb{N}}$ is a standard sequence of mollifiers. In particular, since $F_{n} \in C^{\infty}$, then $f_{n} \in C^{\infty}(\Xi) \subset L^{\infty}(\Xi)$. Recalling that $\nabla\left(\zeta_{n} * F\right)=\zeta_{n} * \nabla F$, we have

$$
\left|f_{n}-f\right|_{-1}^{2}=\left|\nabla\left(F_{n}-F\right)\right|_{2}^{2}=\left|\zeta_{n} *(\nabla F)-\nabla F\right|_{2}^{2} \rightarrow 0
$$

as $n \rightarrow \infty$ because $\nabla F \in L^{2}$ and $\zeta_{n} * \phi \rightarrow \phi$ in $L^{2}$ for all $\phi \in L^{2}$. Moreover, Young's inequality for convolutions yields

$$
\left|f_{n}\right|_{-1}=\left|\zeta_{n} * \nabla F\right|_{2} \leq\left|\zeta_{n}\right|_{1}|\nabla F|_{2}=|\nabla F|_{2}=|f|_{-1} .
$$

The map associating $f$ to $f_{n}$ will de noted by $\Lambda_{n}$.

Proof of theorem 6.2. Uniqueness follows by (4.7). In fact, the estimate is stable with respect to passage to the limit in $G_{1}$ and $G_{2}$.

For fixed $s \in[0, T]$ and $\kappa \in Q_{M}^{1 / 2} K$ we have $G(s) \kappa \in H^{-1}$, and we define $G_{n}(s) \kappa:=$ $\Lambda_{n} G(s) \kappa$. Let us show that

$$
\lim _{n \rightarrow \infty} \mathbb{E} \int_{0}^{T}\left|G_{n}(s)-G(s)\right|_{Q_{M}}^{2} d\langle M\rangle(s)=0 .
$$

The claim follows by (6.2), the dominated convergence theorem and the inequality

$$
\left|G_{n}(s) Q_{M}^{1 / 2} e_{k}\right|_{-1} \leq\left|G(s) Q_{M}^{1 / 2} e_{k}\right|_{-1} \quad \mathbb{P} \text {-a.s. }
$$


which holds for all $s \in[0, T]$ and all $k \in \mathbb{N}$, where $\left(e_{k}\right)_{k \in \mathbb{N}}$ is a basis of $K$, as it follows by (6.3).

Now (4.7) implies that

$$
\sup _{t \leq T} \mathbb{E}\left|Y_{n}(t)-Y_{m}(t)\right|_{-1}^{2} \leq \mathbb{E} \int_{0}^{T}\left|G_{n}(s)-G_{m}(s)\right|_{Q_{M}}^{2} d\langle M\rangle(s),
$$

that is $\left\{Y_{n}\right\}_{n \in \mathbb{N}}$ is a Cauchy sequence in $\mathcal{H}_{2}(T)$, which converges to a $\mathcal{H}$-generalized solution $Y$ of (2.8).

Let us show that $\left\{Y_{n}\right\}_{n \in \mathbb{N}}$ is a Cauchy sequence also in $\mathbb{H}_{2}(T)$, which proves the existence of a $\mathbb{H}$-generalized solution. In fact, using an argument based on Itô's formula and Davis' inequality completely analogous to that leading to (5.4), we obtain

$$
\mathbb{E} \sup _{t \leq T}\left|Y_{n}(t)-Y_{m}(t)\right|^{2} \leq N \mathbb{E} \int_{0}^{T}\left|G_{n}(t)-G_{m}(t)\right|_{Q_{M}}^{2} d\langle M\rangle(t),
$$

where $N$ is a positive constant.

It is now possible to extend the result to equations with multiplicative noise.

Theorem 6.3. Assume that $M$ has stationary independent increments and $B$ is as in (6.1). Then (2.3) admits a unique generalized solution. Moreover, the solution map $x \mapsto X$ is Lipschitz from $\mathcal{H}_{2}$ to $\mathbb{H}_{2}(T)$.

Proof. The argument is an extension of that used in the proof of theorem 2.4, using the previous theorem. In fact, let $X \in \mathbb{H}_{2}(T)$ and consider equation (5.1), which admits a unique generalized solution by theorem 6.2. Since estimates (5.3) and (5.4) hold also for generalized solutions (by a now obvious limiting procedure), the map associating $X$ to $Y$, as defined in the proof of theorem 2.4, is a contraction in $\mathbb{H}_{2}\left(T_{0}\right)$ for a suitably small $T_{0}$. The rest of the proof is identical to that of theorem 2.4

\section{Acknowledgments}

The second named author is partially supported by the Sonderforschungsbereich 611 (Bonn), and by the Centre de Recerca Matemàtica (Barcelona) through an IPDE fellowship.

\section{References}

[1] V. Barbu, Nonlinear semigroups and differential equations in Banach spaces, Noordhoff, Leyden, 1976. MR MR0390843 (52 \#11666)

[2] V. Barbu, G. Da Prato, and M. Röckner, Existence of strong solutions for stochastic porous media equations under general monotonicity conditions, Ann. Probab. (to appear).

[3] P. Benilan and H. Brézis, Solutions faibles d'équations d'évolution dans les espaces de Hilbert, Ann. Inst. Fourier (Grenoble) 22 (1972), no. 2, 311-329. MR MR0336471 (49 \#1245)

[4] H. Brézis, Opérateurs maximaux monotones et semi-groupes de contractions dans les espaces de Hilbert, North-Holland Publishing Co., Amsterdam, 1973. MR MR0348562 (50 \#1060) 
[5] G. Da Prato, Kolmogorov equations for stochastic PDEs, Birkhäuser Verlag, Basel, 2004.

[6] G. Da Prato and J. Zabczyk, Stochastic equations in infinite dimensions, Cambridge UP, 1992.

[7] I. Gyöngy, On stochastic equations with respect to semimartingales. III, Stochastics 7 (1982), no. 4, 231-254.

[8] J. U. Kim, On the stochastic porous medium equation, J. Differential Equations 220 (2006), no. 1, 163-194. MR MR2182084 (2006i:35188)

[9] N. V. Krylov and B. L. Rozovskiu, Stochastic evolution equations, Current problems in mathematics, Vol. 14 (Russian), Akad. Nauk SSSR, Vsesoyuz. Inst. Nauchn. i Tekhn. Informatsii, Moscow, 1979, pp. 71-147, 256. MR MR570795 (81m:60116)

[10] M. Métivier, Semimartingales, Walter de Gruyter \& Co., Berlin, 1982. MR MR688144 (84i:60002)

[11] M. Métivier and G. Pistone, Une formule d'isométrie pour l'intégrale stochastique hilbertienne et équations d'évolution linéaires stochastiques, Z. Wahrscheinlichkeitstheorie und Verw. Gebiete 33 (1975/76), no. 1, 1-18. MR MR0383527 (52 \#4408)

[12] _ Sur une équation d'évolution stochastique, Bull. Soc. Math. France 104 (1976), no. 1, 65-85. MR MR0420854 (54 \#8866)

[13] Sz. Peszat and J. Zabczyk, Stochastic partial differential equations with Lévy noise, Cambridge University Press, Cambridge, 2007. MR MR2356959

[14] C. Prévôt and M. Röckner, A concise course on stochastic partial differential equations, Lecture Notes in Mathematics, vol. 1905, Springer, Berlin, 2007. MR MR2329435

[15] J. Ren, M. Röckner, and F.-Y. Wang, Stochastic generalized porous media and fast diffusion equations, J. Differential Equations 238 (2007), no. 1, 118-152. MR MR2334594

[16] J. L. Vázquez, The porous medium equation, Oxford University Press, Oxford, 2007. MR MR2286292 